\title{
P32 Nationwide Comprehensive Health Protection Screening Program in Hungary 2010-2020-2030
}

\author{
István Barna ${ }^{1, *}$, Ede Kékes ${ }^{2}$, Gergely Dankovics ${ }^{3}$, Tennó Daiki ${ }^{4}$ \\ ${ }^{1}$ Semmelweis University, Faculty of Medicine. I. Department of Internal Medicine, Hungary \\ ${ }^{2}$ Cardiology, University of Pécs Medical School, Pécs, Hungary \\ ${ }^{3}$ MAESZ Program, Szentendre, Hungary \\ ${ }^{4}$ Eötvös Loránd University, Budapest, Hungary
}

\begin{abstract}
From 2010 a new, complex screening program started in Hungary, it works with the support of 76 professional-scientific societies and companies. Within the framework of the Program in the largest mobile diagnostics center in Hungary 37 comprehensive surveys in free form. This enormous special screening station allows 5,100 tests to be performed at each of the 200 screening stations nationwide. The screening program for over 20 years provides 15 million trials on 3,000 scenes and 1 million adult visitors in free humanitarian form. The special screening lorry visited 1696 places, 206,529 inhabitants had a complex screen and 500,395 individuals received information packages about the right lifestyle, the prevention of illnesses and preservation of health and we have received 14 million lifestyle question answers on sites. Based on data from 2018, the age of participants was 42 years for women and 40 years in men. $22 \%$ in women, $23 \%$ in men was the rate of known hypertension and $5.1 \%$ and $4.5 \%$ was the proportion of known diabetics irrespectively. Similarly, the frequency of the known heart and kidney disease was low, but asthma allergy and eye disease were present in more than $20 \%$. Myocardial infarction $26-22 \%$, hypertension disease $62-55 \%$, stroke $19-14 \%$, cancer $47-38 \%$ and metabolic disease $42-34 \%$ occurred in the family history. BMI was increased in both genders, $45 \%$ in women and $59 \%$ in men. In both sexes, the proportion of smokers was over $24 \%$. Based on the results, it has been repeatedly found that the Hungarian population has high risk.
\end{abstract}

(C) 2019 Association for Research into Arterial Structure and Physiology. Publishing services by Atlantis Press International B.V. This is an open access article distributed under the CC BY-NC 4.0 license (http://creativecommons.org/licenses/by-nc/4.0/). 